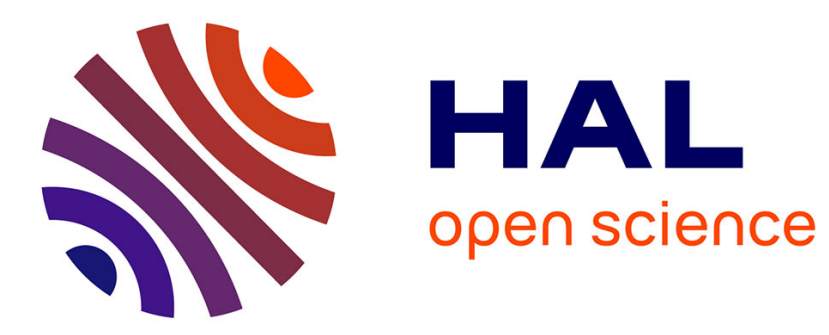

\title{
An axiomatic approach to support in argumentation
}

\author{
Claudette Cayrol, Marie-Christine Lagasquie-Schiex
}

\section{To cite this version:}

Claudette Cayrol, Marie-Christine Lagasquie-Schiex. An axiomatic approach to support in argumentation. 3rd International Workshop on Theory and Applications of Formal Argument (TAFA 2015), Jul 2015, Buenos Aires, Argentina. pp.74-91, 10.1007/978-3-319-28460-6_5 . hal-03198233

\section{HAL Id: hal-03198233 \\ https://hal.science/hal-03198233}

Submitted on 14 Apr 2021

HAL is a multi-disciplinary open access archive for the deposit and dissemination of scientific research documents, whether they are published or not. The documents may come from teaching and research institutions in France or abroad, or from public or private research centers.
L'archive ouverte pluridisciplinaire HAL, est destinée au dépôt et à la diffusion de documents scientifiques de niveau recherche, publiés ou non, émanant des établissements d'enseignement et de recherche français ou étrangers, des laboratoires publics ou privés. 


\title{
An axiomatic approach to support in argumentation
}

\author{
Claudette Cayrol and Marie-Christine Lagasquie-Schiex \\ IRIT-UPS, Toulouse, France \\ \{ccayrol, lagasq\}@irit.fr
}

\begin{abstract}
In the context of bipolar argumentation (argumentation with two kinds of interaction, attacks and supports), we present an axiomatic approach for taking into account a special interpretation of the support relation, the necessary support. We propose constraints that should be imposed to a bipolar argumentation system using this interpretation. Some of these constraints concern the new attack relations, others concern acceptability. We extend basic Dung's framework in different ways in order to propose frameworks suitable for encoding these constraints. By the way, we propose a formal study of properties of necessary support.
\end{abstract}

Keywords: abstract argumentation, bipolar argumentation, axiomatization of necessary support

\section{Introduction}

The main feature of argumentation framework is the ability to deal with incomplete and / or contradictory information, especially for reasoning [15; 2]. Moreover, argumentation can be used to formalize dialogues between several agents by modeling the exchange of arguments in, e.g., negotiation between agents [4]. An argumentation system (AS) consists of a collection of arguments interacting with each other through a relation reflecting conflicts between them, called attack. The issue of argumentation is then to determine "acceptable" sets of arguments (i.e., sets able to defend themselves collectively while avoiding internal attacks), called "extensions", and thus to reach a coherent conclusion. Formal frameworks have greatly eased the modeling and study of AS. In particular, the framework of [15] allows for abstracting the "concrete" meaning of the arguments and relies only on binary interactions that may exist between them.

In this paper, we are interested in bipolar AS (BAS), which handle a second kind of interaction, the support relation. This relation represents a positive interaction between arguments and has been first introduced by [18; 27]. In [8], the support relation is left general so that the bipolar framework keeps a high level of abstraction. However there is no single interpretation of the support, and a number of researchers proposed specialized variants of the support relation: deductive support [5], necessary support [21; 22], evidential support [23; 24], backing support [13]. Each specialization can be associated with an appropriate modelling using an appropriate complex attack. These proposals have been developed quite independently, based on different intuitions and with different formalizations. [10] presents a comparative study in order to restate these proposals in a common setting, the bipolar argumentation framework (see also [13] 
for another survey). The idea is to keep the original arguments, to add complex attacks defined by the combination of the original attack and the support, and to modify the classical notions of acceptability. An important result of [10] is the highlight of a kind of duality between the deductive and the necessary specialization of support, which results in a duality in the modelling by complex attacks. In this context, new different papers have recently been written: some of them give a translation between necessary supports and evidential supports [25]; others propose a justification of the necessary support using the notion of subarguments [26]; an extension of the necessary support is presented in [20]. From all these works it seems interesting to focus on the necessary support. However, different interpretations remain possible, leading to different ways of introducing new attacks and different ways to define acceptability of sets of arguments.

Our purpose is to propose a kind of "axiomatic approach" for studying how necessary support should be taken into account. Indeed we propose requirements (or constraints) that should be imposed to a bipolar argumentation system as "axioms" describing a desired behaviour of this system. Some of these constraints concern the new attack relations, others concern acceptability. We extend basic Dung's framework in different ways in order to propose frameworks suitable for encoding these contraints. By the way, we propose a formal study of properties of necessary support.

Some background is given in Section 2 for AS and BAS, in particular the duality identified in [10]. Section 3 presents constraints that should be imposed for taking into account necessary support. Then different frameworks for handling these constraints are described in Section 4. Section 5 concludes and suggests perspectives of our work. The proofs are given in [11].

\section{Background on abstract bipolar argumentation systems}

Bipolar abstract argumentation systems extend Dung's argumentation systems. So first we recall Dung's framework for abstract argumentation systems.

\subsection{Dung's framework}

Dung's abstract framework consists of a set of arguments and only one type of interaction between them, namely attack. The important point is the way arguments are in conflict.

Def. 1 (Dung AS) A Dung's argumentation system (AS, for short) is a pair $\langle\mathbf{A}, \mathbf{R}\rangle$ where $\mathbf{A}$ is a finite and non-empty set of arguments and $\mathbf{R}$ is a binary relation over $\mathbf{A}$ (a subset of $\mathbf{A} \times \mathbf{A}$ ), called the attack relation.

An argumentation system can be represented by a directed graph, called the interaction graph, in which nodes represent arguments and edges are defined by the attack relation: $\forall a, b \in \mathbf{A}, a \mathbf{R} b$ is represented by $a \not \rightarrow b$.

Def. 2 (Admissibility in AS) Given $\langle\mathbf{A}, \mathbf{R}\rangle$ and $S \subseteq \mathbf{A} . \quad S$ is conflict-free in $\langle\mathbf{A}, \mathbf{R}\rangle$ if and only if (iff, for short) there are no arguments $a, b \in S$, such that (s.t., for short) $a \mathbf{R} b . a \in \mathbf{A}$ is acceptable in $\langle\mathbf{A}, \mathbf{R}\rangle$ with respect to (wrt, for short) $S$ iff $\forall b \in \mathbf{A}$ s.t. $b \mathbf{R} a, \exists c \in S$ s.t. $c \mathbf{R} b$. $S$ is admissible in $\langle\mathbf{A}, \mathbf{R}\rangle$ iff $S$ is conflict-free and each argument in $S$ is acceptable wrt $S$. 
Standard semantics introduced by Dung (preferred, stable, grounded) enable to characterize admissible sets of arguments that satisfy some form of optimality.

Def. 3 (Extensions) Given $\langle\mathbf{A}, \mathbf{R}\rangle$ and $S \subseteq$ A. $S$ is a preferred extension of $\langle\mathbf{A}, \mathbf{R}\rangle$ iff it is a maximal (wrt $\subseteq$ ) admissible set. $S$ is a stable extension of $\langle\mathbf{A}, \mathbf{R}\rangle$ iff it is conflict-free and for each $a \notin S$, there is $b \in S$ s.t. $b \mathbf{R} a . S$ is the grounded extension of $\langle\mathbf{A}, \mathbf{R}\rangle$ iff it is the least (wrt $\subseteq$ ) admissible set $X$ s.t. each argument acceptable wrt $X$ belongs to $X$.

Ex. 1 Let AS be defined by $\mathbf{A}=\{a, b, c, d, e\}$ and $\mathbf{R}=\{(a, b),(b, a),(b, c)$, $(c, d),(d, e),(e, c)\}$. There are two preferred extensions $(\{a\}$ and $\{b, d\})$, one stable extension $(\{b, d\})$ and the grounded extension is the empty set.

\subsection{Abstract bipolar argumentation systems}

The abstract bipolar argumentation framework presented in [8;9] extends Dung's framework in order to take into account both negative interactions expressed by the attack relation and positive interactions expressed by a support relation (see [3] for a more general survey about bipolarity in argumentation).

Def. 4 (BAS) A bipolar argumentation system (BAS, for short) is a tuple $\left\langle\mathbf{A}, \mathbf{R}_{\text {att }}, \mathbf{R}_{\text {sup }}\right\rangle$ where $\mathbf{A}$ is a finite and non-empty set of arguments, $\mathbf{R}_{\text {att }}$ is a binary relation over $\mathbf{A}$ called the attack relation and $\mathbf{R}_{\text {sup }}$ is a binary relation over $\mathbf{A}$ called the support relation.

A BAS can still be represented by a directed graph ${ }^{1}$, called the bipolar interaction graph, with two kinds of edges. Let $a_{i}$ and $a_{j} \in \mathbf{A}, a_{i} \mathbf{R}_{\text {att }} a_{j}$ (resp. $a_{i} \mathbf{R}_{\text {sup }} a_{j}$ ) means that $a_{i}$ attacks $a_{j}$ (resp. $a_{i}$ supports $a_{j}$ ) and it is represented by $a \nrightarrow \rightarrow b$ (resp. $a \rightarrow b$ ).

Handling support and attack at an abstract level has the advantage to keep genericity. An abstract bipolar framework is useful as an analytic tool for studying different notions of complex attacks, complex conflicts, and new semantics taking into account both kinds of interactions between arguments. However, the drawback is the lack of guidelines for choosing the appropriate definitions and semantics depending on the application. For solving this problem, some specializations of the support relation have been proposed and discussed recently. The distinction between deductive and necessary support has appeared first. Then, several interpretations have been given to the necessary support (sub-argument relation [26], evidential support [23; 24; 25], backing support [13]).

Deductive support The deductive support has first appeared in [5]. This variant is intended to enforce the following constraint: If $b \mathbf{R}_{\text {sup }} c$ then "the acceptance of $b$ implies the acceptance of $c$ ", and as a consequence "the non-acceptance of $c$ implies the non-acceptance of $b$ ".

In relevant literature, this interpretation is usually taken into account by adding two kinds of complex attack. The idea is to produce a new AS, containing original and new attacks, and then to use standard semantics.

1 This is an abuse of language since, stricly speaking, this is an edge-labeled graph (with two labels) rather than a directed graph. 
The first new attack, called mediated attack in [5], occurs when $b \mathbf{R}_{\text {sup }} c$ and $a \mathbf{R}_{\text {att }} c$ : "the acceptance of $a$ implies the non-acceptance of $c$ " and so "the acceptance of $a$ implies the non-acceptance of $b$ ". Another complex attack, called supported attacks in [9] occurs when $a \mathbf{R}_{\text {sup }} c$ and $c \mathbf{R}_{\text {att }} b$ : "the acceptance of $a$ implies the acceptance of $c$ " and "the acceptance of $c$ implies the non-acceptance of $b$ "; so, "the acceptance of $a$ implies the non-acceptance of $b$ ".

Def. 5 ([5] Mediated attack, [9] Supported attack)

Let $\mathrm{BAS}=\left\langle\mathbf{A}, \mathbf{R}_{\mathrm{att}}, \mathbf{R}_{\mathrm{sup}}\right\rangle$. There is a mediated attack from a to $b$ iff there is a sequence $a_{1} \mathbf{R}_{\text {sup }} \ldots \mathbf{R}_{\text {sup }} a_{n-1}$, and $a_{n} \mathbf{R}_{\text {att }} a_{n-1}, n \geq 3$, with $a_{1}=b, a_{n}=a$. There is a supported attack from a to $b$ iff there is a sequence $a_{1} \mathbf{R}_{1} \ldots \mathbf{R}_{n-1} a_{n}$, $n \geq 3$, with $a_{1}=a, a_{n}=b, \forall i=1 \ldots n-2, \mathbf{R}_{i}=\mathbf{R}_{\text {sup }}$ and $\mathbf{R}_{n-1}=\mathbf{R}_{\text {att }}$.

So, with the deductive interpretation of the support, new kinds of attack, from $a$ to $b$, can be considered in the following cases:

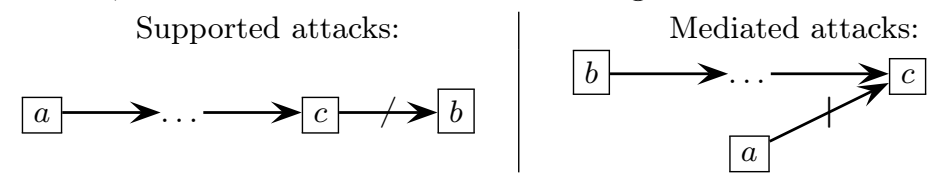

Necessary support The necessary support has been first proposed by [21; 22 ] with the following interpretation: If $c \mathbf{R}_{\text {sup }} b$ then "the acceptance of $c$ is necessary to get the acceptance of $b$ ", or equivalently "the acceptance of $b$ implies the acceptance of $c "$. A example of this kind of support could be:

Ex. 2 A dialog between three customers about the qualities of services of their hotel:

- "This hotel is very well managed." (Argument a)

- "Yes. In particular, the hotel staff is very competent." (Argument b)

- "They are not competent! The rooms are dirty." (Argument c)

Here $b$ necessarily supports $a$ and $c$ attacks $b(c \not \rightarrow b \rightarrow a)$. The link between $b$ and $a$ is similar to the notion of subargument used in [26].

As for deductive support, the idea is to add complex attacks in order to use standard semantics on a new AS. The first added complex attack, called extended attack in [21] and secondary attack in [9] has been proposed in the following case: Suppose that $a \mathbf{R}_{\text {att }} c$ and $c \mathbf{R}_{\text {sup }} b$. "The acceptance of $a$ implies the non-acceptance of $c$ " and so "the acceptance of $a$ implies the non-acceptance of $b$ ". Another kind of complex attack may be considered when $c \mathbf{R}_{\text {sup }} a$ and $c \mathbf{R}_{\text {att }} b$ : "the acceptance of $a$ implies the acceptance of $c$ " and "the acceptance of $c$ implies the non-acceptance of $b$ ". So, "the acceptance of $a$ implies the non-acceptance of $b$ ". This new attack from $a$ to $b$ has been proposed in [22].

The formal definition of these two attacks is:

Def. 6 ([22] Extended attack) Let BAS $=\left\langle\mathbf{A}, \mathbf{R}_{\mathrm{att}}, \mathbf{R}_{\mathrm{sup}}\right\rangle$. There is an extended attack from a to $b$ iff either $a \mathbf{R}_{\text {att }} b$ (direct attack), or there is a sequence $a_{1} \mathbf{R}_{\text {att }} a_{2} \mathbf{R}_{\text {sup }} \ldots \mathbf{R}_{\text {sup }} a_{n}, n \geq 3$, with $a_{1}=a, a_{n}=b$ (Case 1$)$, or there is $a$ sequence $a_{1} \mathbf{R}_{\text {sup }} \ldots \mathbf{R}_{\text {sup }} a_{n}$, and $a_{1} \mathbf{R}_{\text {att }} a_{p}, n \geq 2$, with $a_{n}=a, a_{p}=b$ (Case 2).

So, with the necessary interpretation of the support, new kinds of attack, from $a$ to $b$, can be considered in the following cases: 


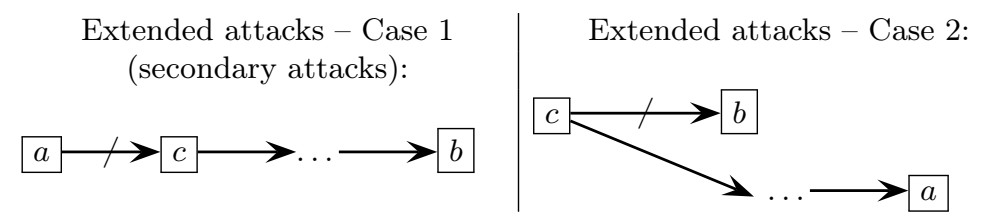

Duality between deductive and necessary support Deductive support and necessary support have been introduced independently. Nevertheless, they correspond to dual interpretations of the notion of support. Let us denote $a \stackrel{D}{\rightarrow}$ $b$ (resp. $a \stackrel{N}{\rightarrow} b$ ) when there exists a deductive (resp. necessary) support from $a$ to $b$. As $a \stackrel{D}{\rightarrow} b$ means that "the acceptance of $a$ implies the acceptance of $b$ ", and $a \stackrel{N}{\rightarrow} b$ means that "the acceptance of $a$ is necessary to get the acceptance of $b$ ", it follows that $a \stackrel{N}{\rightarrow} b$ is equivalent to $b \stackrel{D}{\rightarrow} a$.

Following this duality, it is easy to see that the mediated attack obtained by combining the attack relation $\mathbf{R}_{\text {att }}$ and the support relation $\mathbf{R}_{\text {sup }}$ exactly corresponds to the secondary attack obtained by combining the attack relation $\mathbf{R}_{\text {att }}$ and the support relation $\mathbf{R}_{\text {sup }}^{-1}$ which is the symmetric relation of $\mathbf{R}_{\text {sup }}\left(\mathbf{R}_{\text {sup }}^{-1}\right.$ $\left.=\left\{(b, a) \mid(a, b) \in \mathbf{R}_{\text {sup }}\right\}\right)$. Similarly, the supported attack obtained by combining the attack relation $\mathbf{R}_{\text {att }}$ and the support relation $\mathbf{R}_{\text {sup }}$ exactly corresponds to the second case of extended attack obtained by combining the attack relation $\mathbf{R}_{\text {att }}$ and the support relation $\mathbf{R}_{\text {sup }}^{-1}$.

So in the following, we only focus on the necessary support since, taking advantage of the duality, all the results we obtain can be easily translated into results for deductive supports.

\section{Axiomatic approach for handling necessary support}

In relevant literature, as described in the previous section, taking into account support generally leads to add new attacks. It is the case for instance with the necessary support that leads to extended attacks. However, a deeper analysis of the original interpretation of necessary support suggests other ways to handle this support. In this section, we discuss several constraints induced by the intended meaning of necessary support, and we show that new frameworks must be proposed for encoding these constraints.

Let us come back to the original interpretation of necessary support: If $c \mathbf{R}_{\text {sup }} b$, "the acceptance of $c$ is necessary to get the acceptance of $b$ ". Analysing this interpretation leads to at least four kinds of constraints.

Transitivity (TRA) This first requirement concerns the relation $\mathbf{R}_{\text {sup }}$ alone. It expresses transitivity ${ }^{2}$ of the necessary support. It induces that a sequence of supports is considered as a support:

Def. 7 (Constraint TRA) $\forall a, b \in \mathbf{A}$, if $\exists n>1$ such that $a=a_{1} \mathbf{R}_{\text {sup }}$ $\ldots \mathbf{R}_{\mathrm{sup}} a_{n}=b$, then $a$ supports $b$.

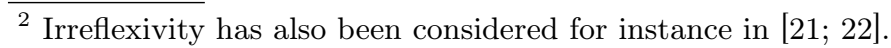


Closure (CLO) A second constraint also concerns the relation $\mathbf{R}_{\text {sup }}$ alone and expresses the fact that if $c \mathbf{R}_{\text {sup }} b$, then "the acceptance of $b$ implies the acceptance of $c$ ". So, if $c \mathbf{R}_{\text {sup }} b$, and there exists an extension $S$ containing $b$, then $S$ also contains $c$. This constraint can be expressed by the property of closure of an extension under $\mathbf{R}_{\text {sup }}^{-1}:^{3}$

Def. 8 (Constraint CLO) Let $s$ be a semantics and $E$ be an extension under $s . \forall a, b \in \mathbf{A}$, if $a \mathbf{R}_{\text {sup }} b$ and $b \in E$, then $a \in E$.

Moreover, an interesting variant of this constraint could be induced by a slightly different reading of the original interpretation: "the acceptance of $c$ is necessary to get the acceptance of $b$ " because $c$ is the only attacker of a particular attacker of $b$. This reading implies that there implicitly exists a special attack to $b$ which can be only defeated by $c$. This interpretation will lead us to propose a framework with meta-arguments (see Section 4.2).

Conflicting sets (CFS) Now, we consider constraints induced by the presence of both attacks and supports in a BAS. Starting from the original interpretation, if $a \mathbf{R}_{\text {att }} c$ and $c \mathbf{R}_{\text {sup }} b$, "the acceptance of $a$ implies the non-acceptance of $c$ " and "the acceptance of $b$ implies the acceptance of $c$ ". So, using contrapositives, "the acceptance of $a$ implies the non-acceptance of $b$ ", and then "the acceptance of $b$ implies the non-acceptance of $a$ ". Thus, we obtain a symmetric constraint involving $a$ and $b$. However, the fact that "the acceptance of $a$ implies the non-acceptance of $b$ " is not equivalent to the fact that there is an attack from $a$ to $b$. We have only the sufficient condition. So, the creation of a complex attack (here a secondary attack) from $a$ to $b$ can be viewed in some sense too strong. Hence, faced with the case when $a \mathbf{R}_{\text {att }} c$ and $c \mathbf{R}_{\text {sup }} b$, we propose to assert a conflict between $a$ and $b$, or in other words that the set $\{a, b\}$ is a conflicting set. Similarly, if $c \mathbf{R}_{\text {att }} b$ and $c \mathbf{R}_{\text {sup }} a$, "the acceptance of $a$ implies the acceptance of $c$ " and so "the acceptance of $a$ implies the non-acceptance of $b$ ".

Def. 9 (Constraint CFS) $\forall a, b, c \in \mathbf{A}$. If ( $a \mathbf{R}_{\text {att }} c$ and $c$ supports $b$ ) or $\left(c \mathbf{R}_{\text {att }} b\right.$ and $c$ supports $\left.a\right)$ then $\{a, b\}$ is a conflicting set.

Note that the Dung's abstract framework is not suitable for expressing such a constraint. So we will present in Section 4.1 a new framework for handling conflicting sets of arguments.

Addition of new attacks (nATT and n+ATT) Beyond these properties, according to the applications and the previous works presented in literature, we may impose stronger constraints corresponding to the addition of new attacks. Two cases may be considered:

Def. 10 (Constraint $\mathbf{n A T T}$ ) If $a \mathbf{R}_{\text {att }} c$ and $c \mathbf{R}_{\text {sup }} b$, then there is a new attack from a to $b$.

Def. 11 (Constraint $\mathbf{n}+\mathbf{A T T})$ If $\left(a \mathbf{R}_{\mathrm{att}} c\right.$ and $\left.c \mathbf{R}_{\text {sup }} b\right)$ or $\left(c \mathbf{R}_{\text {att }} b\right.$ and $\left.c \mathbf{R}_{\text {sup }} a\right)$, then there is a new attack from a to $b$.

\footnotetext{
${ }^{3}$ Note that if $c \mathbf{R}_{\text {sup }} b$ and $c \mathbf{R}_{\text {att }} b$, as an extension must be conflict-free, there is no extension containing both $c$ and $b$, so the constraint trivially holds. Some works, as for instance [10], exclude the case when $c \mathbf{R}_{\text {sup }} b$ and $c \mathbf{R}_{\text {att }} b$.
} 
nATT (resp. $\mathbf{n}+\mathbf{A T T}$ ) corresponds to the addition of secondary (resp. extended) attacks. In Section 4.3 we present two frameworks for handling these constraints.

Continuing the discussion one step further, if the fact that "the acceptance of $a$ implies the non-acceptance of $b$ " is represented by an attack from $a$ to $b$, due to contrapositive, this new attack must be symmetric. However, in that case, each attack should be turned into a symmetric one. Thus, we move towards symmetric argumentation frameworks which have been studied in [14]. We will not consider this case in the current paper. Some of the above constraints can he handled in a Dung's abstract framework (CLO, TRA, nATT and n+ATT) with the advantage of reusing all known Dung's results. However, as we noticed above, constraint CFS cannot be encoded in a Dung's framework. So in the next section we propose different variants of Dung's framework and of the bipolar framework in order to take into account these constraints.

\section{New frameworks for handling necessary supports}

Starting from the constraints discussed in Section 3, we propose several frameworks for handling necessary support. The first two are driven by Constraint CLO whereas the last two are driven by the constraints nATT and $\mathbf{n}+\mathbf{A T T}$. The section will end by a comparison of these frameworks.

\subsection{Handling conflicting sets of arguments}

We propose a generalized bipolar abstract argumentation framework consisting of a set of arguments, a binary relation representing an attack between arguments, a binary relation representing a support between arguments and a set of conflicting sets of arguments. Intuitively, knowing that $a$ attacks $b$ is stronger than knowing that $\{a, b\}$ is a conflicting set of arguments. Knowing that a set of arguments $S$ is conflicting will only prevent any extension from containing $S$. Moreover, a conflicting set may contain more than two arguments.

Def. 12 (Generalized BAS, GBAS) A generalized bipolar argumentation system is a tuple $\left\langle\mathbf{A}, \mathbf{R}_{\text {att }}, \mathbf{R}_{\text {sup }}, \mathbf{C}\right\rangle$ where $\mathbf{A}$ is a finite and non-empty set of arguments, $\mathbf{R}_{\text {att }}$ is a binary relation over $\mathbf{A}$ called the attack relation, $\mathbf{R}_{\text {sup }}$ is a binary relation over $\mathbf{A}$ called the support relation and $\mathbf{C}$ is a finite set of subsets of $\mathbf{A}$ such that $\forall(a, b) \in \mathbf{R}_{\text {att }},\{a, b\} \in \mathbf{C}$.

Conflict-freeness in a generalized bipolar argumentation system is defined as follows:

Def. 13 (Conflict-freeness in a GBAS) Let $\left\langle\mathbf{A}, \mathbf{R}_{\text {att }}, \mathbf{R}_{\text {sup }}, \mathbf{C}\right\rangle$ be a $G B A S$ and $S \subseteq \mathbf{A} . S$ is conflict-free in the $G B A S$ iff there does not exist $C \in \mathbf{C}$ such that $C \subseteq S$.

However, the definition of semantics depends on the interpretation of the support and also on the constraints that have to be enforced. The generalized bipolar framework can be instantiated for encoding necessary support, due to the following definition: 
Def. 14 Let $\mathrm{BAS}=\left\langle\mathbf{A}, \mathbf{R}_{\text {att }}, \mathbf{R}_{\text {sup }}\right\rangle$ with $\mathbf{R}_{\text {sup }}$ being a set of necessary supports. The tuple GBAS $=\left\langle\mathbf{A}, \mathbf{R}_{\text {att }}, \mathbf{R}_{\text {sup }}, \mathbf{C}\right\rangle$ with $\mathbf{C}=\left\{\{a, b\} \mid(a, b) \in \mathbf{R}_{\text {att }}\right\} \cup$ $\left\{\{a, b\} \mid a \mathbf{R}_{\text {att }} c\right.$ and $c$ supports $\left.b\right\} \cup\left\{\{a, b\} \mid c \mathbf{R}_{\text {att }} b\right.$ and $c$ supports $\left.a\right\}$ is the generalized argumentation system associated with BAS.

It is easy to see that the generalized argumentation system associated with BAS enables to enforce the constraints TRA and CFS, whereas it satisfies neither Constraint nATT, nor Constraint $\mathbf{n}+\mathbf{A T T}$.

The next step is the study of acceptability in a GBAS in order to check whether Contraint CLO is taken into account. For that purpose, the first proposal is to use conflict-freeness as defined in Def. 13 and admissible, preferred and stable extensions as defined in Dung's systems. In this case, it can be proved that every stable extension is closed under $\mathbf{R}_{\text {sup }}^{-1}$.

Prop. 1 Let $\mathrm{BAS}=\left\langle\mathbf{A}, \mathbf{R}_{\text {att }}, \mathbf{R}_{\text {sup }}\right\rangle$ and its associated GBAS. Let $S \subseteq \mathbf{A}$. If $S$ is conflict-free in GBAS, and for each $a \notin S$, there is $b \in S$ s.t. $b \mathbf{R}_{\text {att }} a$, then $S$ is closed under $\mathbf{R}_{\text {sup }}^{-1}$.

However, this approach produces many conflicts, without adding any attacks. So in many cases, there will be no stable extension. Moreover, Constraint CLO is generally not satisfied with the preferred semantics. The following example illustrates these two drawbacks.

Ex. 3 Consider BAS represented by the following graph. $\mathbf{C}=\{\{x, c\},\{x, b\},\{a, c\}\}$. Using the classical definition of

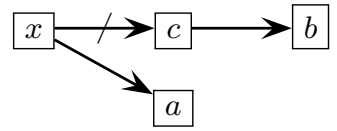
semantics with conflict-freeness as defined in Def. 13, the preferred extensions of the associated GBAS are $\{a, x\}$ and $\{a, b\}$, and there is no stable extension. Moreover, the preferred extension $\{a, b\}$ is not closed under $\mathbf{R}_{\text {sup }}^{-1}$.

The preferred semantics has to be redefined in order to enforce Constraint CLO. So, our second proposal is to enforce a notion of coherence by combining conflict-freeness and closure under $\mathbf{R}_{\text {sup }}^{-1}$. Moreover it can be proven that:

Prop. 2 Let $\left\langle\mathbf{A}, \mathbf{R}_{\text {att }}, \mathbf{R}_{\text {sup }}\right\rangle$ and its associated GBAS. Let $S \subseteq \mathbf{A}$. If $S$ is closed under $\mathbf{R}_{\text {sup }}^{-1}$ then ( $S$ is conflict-free in GBAS iff $S$ is conflict-free in $\left\langle\mathbf{A}, \mathbf{R}_{\text {att }}\right\rangle$ ).

Def. 15 (Coherence in a GBAS) Let $\left\langle\mathbf{A}, \mathbf{R}_{\text {att }}, \mathbf{R}_{\text {sup }}, \mathbf{C}\right\rangle$ be $a G B A S$ and $S \subseteq$ A. $S$ is coherent in the GBAS iff $S$ is conflict-free in $\left\langle\mathbf{A}, \mathbf{R}_{\text {att }}\right\rangle$ and $S$ is closed under $\mathbf{R}_{\text {sup }}^{-1}$.

Using coherence in place of conflict-freeness leads to new definitions:

Def. 16 (Admissibility in a GBAS) Let $\left\langle\mathbf{A}, \mathbf{R}_{\text {att }}, \mathbf{R}_{\text {sup }}, \mathbf{C}\right\rangle$ be $a G B A S$ and $S \subseteq \mathbf{A}$. $S$ is admissible in the GBAS iff $S$ is coherent in the $G B A S$ and $\forall a \in S$, $\forall b \in \mathbf{A}$ s.t. $b \mathbf{R}_{\mathrm{att}} a, \exists c \in S$ s.t. $c \mathbf{R}_{\mathrm{att}} b . S$ is a preferred extension of the GBAS iff it is a maximal (wrt $\subseteq$ ) admissible set. $S$ is a stable extension of the GBAS iff $S$ is coherent $t^{4}$ in the GBAS and for each $a \notin S$, there is $b \in S$ s.t. $b \mathbf{R}_{\text {att }} a$.

\footnotetext{
${ }^{4}$ Due to Prop.1, coherent may be replaced by conflict-free.
} 
Ex.3 (cont'd) Taking into account coherence, as in Def.16, $\{a, x\}$ is the unique preferred extension of the associated GBAS, and it is closed under $\mathbf{R}_{\text {sup }}^{-1}$.

So, using Def.16 and 15, the associated GBAS enables to enforce Constraint CLO ${ }^{5}$ Moreover, as in Dung's framework, stable extensions are also preferred.

Prop. 3 Let $\left\langle\mathbf{A}, \mathbf{R}_{\text {att }}, \mathbf{R}_{\text {sup }}, \mathbf{C}\right\rangle$ be a $G B A S$ and $S \subseteq \mathbf{A}$. If $S$ is a stable extension of the GBAS then $S$ is also a preferred extension of the GBAS.

A thorough study of the generalized bipolar abstract argumentation framework would demand to define other semantics such as grounded one. However, this is not our purpose in this paper. We focus on the way to enforce different kinds of constraints related to necessary support.

\subsection{A meta-framework encoding necessary support}

The fact that "the acceptance of $c$ is necessary to get the acceptance of $b$ " can be encoded in another way. As explained in Section 3, the idea is to assume the existence of a special argument attacking $b$ for which $c$ is the only attacker. More precisely, if $c \mathbf{R}_{\text {sup }} b$, we create a new argument $N_{c b}$ and two attacks $c \mathbf{R}_{\text {att }} N_{c b}$ and $N_{c b} \mathbf{R}_{\text {att }} b$. As $c$ is the unique attacker of $N_{c b}$, "the acceptance of $b$ implies the acceptance of $c$ ". The meaning of $N_{c b}$ could be that the support from $c$ to $b$ is not active. A similar idea can be found in $[28 ; 12]$ for the more general purpose of representing recursive and defeasible attacks and supports.

Def. 17 Let BAS $=\left\langle\mathbf{A}, \mathbf{R}_{\text {att }}, \mathbf{R}_{\text {sup }}\right\rangle$ with $\mathbf{R}_{\text {sup }}$ being a set of necessary supports. Let $\mathbf{A}_{n}=\left\{N_{c b} \mid(c, b) \in \mathbf{R}_{\text {sup }}\right\}$ and $\mathbf{R}_{n}=\left\{\left(c, N_{c b}\right) \mid(c, b) \in \mathbf{R}_{\text {sup }}\right\} \cup$ $\left\{\left(N_{c b}, b\right) \mid(c, b) \in \mathbf{R}_{\text {sup }}\right\}$. The tuple MAS $=\left\langle\mathbf{A} \cup \mathbf{A}_{n}, \mathbf{R}_{\text {att }} \cup \mathbf{R}_{n}\right\rangle$ is the metaargumentation system ${ }^{6}$ associated with $\mathrm{BAS}$.

Let us check whether the minimal requirements are satisfied. Let us first consider constraint TRA. From $a \mathbf{R}_{\text {sup }} b$ and $b \mathbf{R}_{\text {sup }} c$, we obtain the sequence of attacks $a \mathbf{R}_{\text {att }} N_{a b} \mathbf{R}_{\text {att }} b \mathbf{R}_{\text {att }} N_{b c} \mathbf{R}_{\text {att }} c$. So, the acceptance of $c$ implies the acceptance of $b$, which in turn implies the acceptance of $a$, as if we had directly encoded $a \mathbf{R}_{\text {sup }} c$. So, TRA is taken into account. The same result holds for CLO:

Prop. 4 Let $\mathrm{BAS}=\left\langle\mathbf{A}, \mathbf{R}_{\text {att }}, \mathbf{R}_{\text {sup }}\right\rangle$ and its associated MAS. Let $S \subseteq \mathbf{A} \cup \mathbf{A}_{n}$. If $S$ is admissible in MAS, then $S \cap \mathbf{A}$ is closed under $\mathbf{R}_{\text {sup }}^{-1}$ in BAS.

Constraint CFS is not enforced. We only have the following property:

Prop. 5 Let $\mathrm{BAS}=\left\langle\mathbf{A}, \mathbf{R}_{\text {att }}, \mathbf{R}_{\text {sup }}\right\rangle$ and its associated MAS. Let $a, b, c$ be arguments of $\mathbf{A}$. If ( $a \mathbf{R}_{\text {att }} c$ and $c$ supports $\left.b\right)$ or $\left(c \mathbf{R}_{\text {att }} b\right.$ and $c$ supports $\left.a\right)$ then no admissible set in MAS contains $\{a, b\}$.

Note that this result is weaker than CFS since it does not imply that $\{a, b\}$ is a conflicting set.

Obviously, stronger constraints such as $\mathbf{n A T T}$ or $\mathbf{n}+\mathbf{A T T}$ are not directly enforced. If $a \mathbf{R}_{\text {att }} c$ and $c \mathbf{R}_{\text {sup }} b$, we obtain the sequence $a \mathbf{R}_{\text {att }} c \mathbf{R}_{\text {att }} N_{c b} \mathbf{R}_{\text {att }} b$. No attack from $a$ to $b$ is added. However, we will see in Section 4.4 that the metaargumentation framework associated with BAS enables to recover the extensions obtained when enforcing Constraint nATT.

\footnotetext{
${ }^{5}$ Note that enforcing coherence makes the set $C$ useless due to Prop.2.

${ }^{6}$ Note that it is an argumentation system in Dung's sense.
} 


\subsection{A framework with complex attacks}

In this subsection we discuss two frameworks enabling to handle necessary support through the addition of complex attacks. According to the various interpretations of the necessary support, all the complex attacks are not justified. For instance, if the necessary support models a subargument relation as in [26], only the secondary attack makes sense. Other works [22] have considered both cases of extended attack. However, to the best of our knowledge, there has been no formal study of the properties of these extended attacks, and of the consequences of these attacks on the acceptable sets of arguments.

From Def. 6, new attacks called n+-attacks can be generated inductively as follows:

Def. 18 (n+-attacks) Let BAS $=\left\langle\mathbf{A}, \mathbf{R}_{\mathrm{att}}, \mathbf{R}_{\text {sup }}\right\rangle$ with $\mathbf{R}_{\text {sup }}$ being a set of necessary supports. There exists a n+-attack from $a$ to $b$ iff either $a \mathbf{R}_{\text {att }} b$, or there is a (case 1 or case 2) extended attack from a to b, or there exists an argument $c$ s.t. $a \mathrm{n+-attacks} c$ and $c$ supports $b$, or there exists an argument $c$ s.t. $c$ supports $a$ and $c$ n+-attacks $b . \mathbf{N}^{+}{ }_{\mathbf{R}_{\text {att }}}^{\mathbf{R}_{\text {ap }}}$ denoted the set of $\mathrm{n}^{+- \text {attacks }}$ generated by $\mathbf{R}_{\text {sup }}$ on $\mathbf{R}_{\text {att }}$. The AS defined by $\left\langle\mathbf{A}, \mathbf{N}^{+} \mathbf{R}_{\text {att }}^{\mathbf{R}_{\text {ap }}}\right\rangle$ is denoted by $\mathrm{AS}^{N^{+}}$.

Obviously Constraints TRA, nATT and $\mathbf{n}+\mathbf{A T T}$ are enforced in $\mathrm{AS}^{N^{+}}$.

Let us now consider the case when the extended attacks are restricted to secondary attacks (Case 1 of extended attacks). Following the above definition, our purpose is to define a n-attack from $a$ to $b$ when either $a \mathbf{R}_{\text {att }} b$, or there exists a secondary attack from $a$ to $b$, or there exists an argument $c$ s.t. $a$ n-attacks $c$ and $c$ supports $b$. Indeed, it is easy to prove that the formal definition of this $\mathrm{n}$-attack can be simplified as follows:

Def. 19 (n-attack) Let BAS $=\left\langle\mathbf{A}, \mathbf{R}_{\text {att }}, \mathbf{R}_{\text {sup }}\right\rangle$. There is $\mathbf{n}$-attack from a to $b$ iff either $a \mathbf{R}_{\text {att }} b$, or there is a secondary attack from a to $b . \quad \mathbf{N}_{\mathbf{R}_{\text {att }}}^{\mathbf{R}_{\text {au }}}$ denoted the set of $\mathbf{n}$-attacks generated by $\mathbf{R}_{\text {sup }}$ on $\mathbf{R}_{\text {att }}$. The AS defined by $\left\langle\mathbf{A}, \mathbf{N}_{\mathbf{R}_{\text {att }}}^{\mathbf{R}_{\text {ap }}}\right\rangle$ is denoted by $\mathrm{AS}^{N}$.

Note that both $\mathrm{AS}^{N}$ and $\mathrm{AS}^{N^{+}}$are Dung's argumentation systems; so the classical notions given in Def. 2 and 3 can be applied without restriction, nor redefinition.

Obviously Constraints TRA and nATT are enforced in $\mathrm{AS}^{N}$, whereas Constraint $\mathbf{n}+\mathbf{A T T}$ is not.

Def. 18 looks complex. However the following proposition enables to rewrite $\mathrm{n}+$-attacks and $\mathrm{n}$-attacks in a form which will be much easier to handle for studying their properties.

Prop. 6 Let $\mathrm{BAS}=\left\langle\mathbf{A}, \mathbf{R}_{\text {att }}, \mathbf{R}_{\text {sup }}\right\rangle$. There is an $\mathrm{n}+-$ attack from a to $b$ iff there is a sequence $a_{1} \mathbf{R}_{\text {att }} b_{1} \mathbf{R}_{\text {sup }} \ldots \mathbf{R}_{\text {sup }} b_{m}$, with $b_{m}=b$ and $m \geq 1$, and a sequence $a_{1} \mathbf{R}_{\text {sup }} \ldots \mathbf{R}_{\text {sup }} a_{n}$ with $a_{n}=a$ and $n \geq 1$.

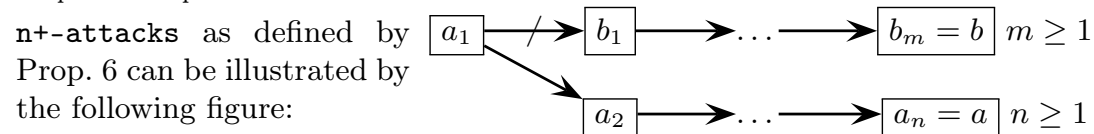

Moreover, Prop. 6 can be used for identifying the following particular cases: 
- The case when $m=n=1$ corresponds to a direct attack from $a$ to $b$.

- The case when $n=1$ and $m \geq 1$ corresponds to a n-attack from $a$ to $b$ (direct or secondary attacks, see Def. 19).

- The case when $n=1$ and $m>1$ corresponds to an extended attack - Case

1 (secondary attack) from $a$ to $b$ (see Def. 6).

- The case when $n>1$ and $m=1$ corresponds to an extended attack - Case

2 from $a$ to $b$ (see Def. 6).

An obvious consequence of this proposition is:

Corol. 1 Let $\mathrm{BAS}=\left\langle\mathbf{A}, \mathbf{R}_{\text {att }}, \mathbf{R}_{\text {sup }}\right\rangle$ and its associated $\mathrm{AS}^{N}$ and $\mathrm{AS}^{N^{+}}$. Let $S \subseteq \mathbf{A}$. If $S$ is conflict-free in $\mathrm{AS}^{N^{+}}$, then $S$ is conflict-free in $\mathrm{AS}^{N}$.

As said above, in some works necessary support can be handled by only considering $\mathrm{n}$-attacks, that is by adding secondary attacks. However, although both cases of extended attacks are independent, we show that taking into account only n-attacks is already enough for inducing constraints on $\mathrm{AS}^{N^{+}}$.

Prop. 7 Let $\mathrm{BAS}=\left\langle\mathbf{A}, \mathbf{R}_{\text {att }}, \mathbf{R}_{\text {sup }}\right\rangle$ and its associated $\mathrm{AS}^{N}$. If a $\mathrm{n}^{+-a t t a c k}$ from $a$ to $b$ can be built from BAS, there exists no admissible set in $\mathrm{AS}^{N}$ containing $\{a, b\}$.

As an immediate consequence (contrapositive of Prop. 7), we have:

Corol. 2 Let $\mathrm{BAS}=\left\langle\mathbf{A}, \mathbf{R}_{\text {att }}, \mathbf{R}_{\text {sup }}\right\rangle$ and the associated $\mathrm{AS}^{N}$ and $\mathrm{AS}^{N^{+}}$. Let $S \subseteq \mathbf{A}$. If $S$ is admissible in $\mathrm{AS}^{N}$, then $S$ is conflict-free in $\mathrm{AS}^{N^{+}}$.

Ex. 4 Consider BAS represented by the following graph:

The associated $\mathrm{AS}^{N}$ only contains the original attack from $c$ to
$b$ (there is no secondary attack). If we consider only $\mathrm{n}$-attacks,
there is no conflict between a and b. However, it can be proved
that no admissible set in $\mathrm{AS}^{N}$ contains $\{a, b\}$.

The following results establish links between extensions in $\mathrm{AS}^{N}$ and $\mathrm{AS}^{N^{+}}$.

Prop. 8 Let $\mathrm{BAS}=\left\langle\mathbf{A}, \mathbf{R}_{\text {att }}, \mathbf{R}_{\text {sup }}\right\rangle$ and the associated $\mathrm{AS}^{N}$ and $\mathrm{AS}^{N^{+}}$. Let $S \subseteq \mathbf{A}$. If $S$ is admissible in $\mathrm{AS}^{N}$, then $S$ is also admissible in $\mathrm{AS}^{N^{+}}$.

The converse of Prop 8 generally does not hold as shown by the following example.

Ex. 5 Consider BAS and its associated $\mathrm{AS}^{N}$ and $\mathrm{AS}^{N^{+}}$represented by the following graphs:

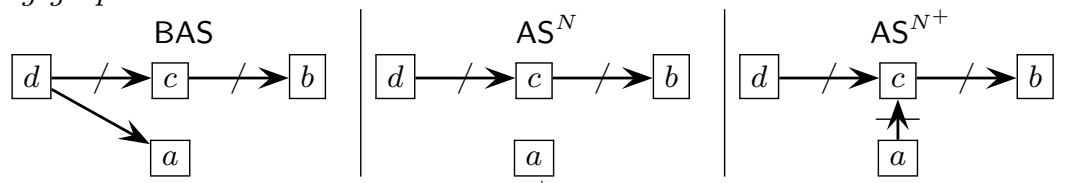

The set $\{a, b\}$ is admissible in $\mathrm{AS}^{N^{+}}$but is not admissible in $\mathrm{AS}^{N}$ (since a does not attack $c$ in $\left.\mathrm{AS}^{N}\right)$.

However, the converse of Prop. 8 holds for maximal admissible sets:

Prop. 9 Let $\mathrm{BAS}=\left\langle\mathbf{A}, \mathbf{R}_{\text {att }}, \mathbf{R}_{\text {sup }}\right\rangle$ and its associated $\mathrm{AS}^{N}$ and $\mathrm{AS}^{N^{+}}$. Let $S \subseteq \mathbf{A} . S$ is maximal admissible in $\mathrm{AS}^{N^{+}}$iff $S$ is maximal admissible in $\mathrm{AS}^{N}$. 
The same holds for stable semantics:

Prop. 10 Let $\mathrm{BAS}=\left\langle\mathbf{A}, \mathbf{R}_{\mathrm{att}}, \mathbf{R}_{\text {sup }}\right\rangle$ and its associated $\mathrm{AS}^{N}$ and $\mathrm{AS}^{N^{+}}$. Let $S \subseteq \mathbf{A} . S$ is stable in $\mathrm{AS}^{N^{+}}$iff $S$ is stable in $\mathrm{AS}^{N}$.

We conclude this section by providing results about the property of closure under the relation $\mathbf{R}_{\text {sup }}^{-1}$.

Prop. 11 Let $\mathrm{BAS}=\left\langle\mathbf{A}, \mathbf{R}_{\text {att }}, \mathbf{R}_{\text {sup }}\right\rangle$ and its associated $\mathrm{AS}^{N^{+}}$. Let $S \subseteq \mathbf{A}$ and $a, b \in \mathbf{A}$. If $S$ is conflict-free in $\mathrm{AS}^{N^{+}}, a \in S$ and $b \mathbf{R}_{\text {sup }} a$, then $S \cup\{b\}$ is conflict-free in $\mathrm{AS}^{N^{+}}$. If $S$ is maximal (wrt $\subseteq$ ) conflict-free in $\mathrm{AS}^{N^{+}}$, then $S$ is closed for the relation $\mathbf{R}_{\text {sup }}^{-1}$.

Prop. 11 does not hold when considering $\mathrm{AS}^{N}$ instead of $\mathrm{AS}^{N^{+}}$, as shown by the following example.

Ex.4 (cont'd) $S=\{a, b\}$ is maximal conflict-free in $\mathrm{AS}^{N}$ but it is not closed under $\mathbf{R}_{\text {sup }}^{-1}$. We have $c \mathbf{R}_{\text {sup }}$ a but $S \cup\{c\}$ is not conflict-free in $\mathrm{AS}^{N}$.

However, the property of closure under $\mathbf{R}_{\text {sup }}^{-1}$ is recovered in $\mathrm{AS}^{N}$, if preferred (resp. stable) extensions are considered.

Prop. 12 Let BAS $=\left\langle\mathbf{A}, \mathbf{R}_{\text {att }}, \mathbf{R}_{\text {sup }}\right\rangle$ and the associated $\mathrm{AS}^{N}$ and $\mathrm{AS}^{N^{+}}$. Let $S \subseteq \mathbf{A}$. If $S$ is a preferred extension in $\mathrm{AS}^{N}$ (resp. $\mathrm{AS}^{N^{+}}$), then $S$ is closed for the relation $\mathbf{R}_{\text {sup }}^{-1}$. If $S$ is stable in $\mathrm{AS}^{N}$ (resp. $\mathrm{AS}^{N^{+}}$), then $S$ is closed for the relation $\mathbf{R}_{\text {sup }}^{-1}$.

Due to Prop. 12, each stable (resp. preferred) extension of $\mathrm{AS}^{N}$ (resp. $\mathrm{AS}^{N^{+}}$) is closed under $\mathbf{R}_{\text {sup }}^{-1}$. In that sense, Constraint $\mathbf{C L O}$ is enforced in $\mathrm{AS}^{N}$ (resp. $\left.\mathrm{AS}^{N^{+}}\right)$.

It remains to consider Constraint CFS. This constraint is obviously satisfied by $\mathrm{AS}^{N^{+}}$since a new attack is built for each conflict in the sense of CFS, whereas the Dung's argumentation system $\mathrm{AS}^{N}$ does not capture all the conflicts induced by CFS, as illustrated by the following example.

Ex.3 (cont'd) In the associated $\mathrm{AS}^{N}$, there is one $\mathrm{n}$-attacks from $x$ to $c$ and one from $x$ to $b .\{a, x\}$ is the unique preferred extension of $\mathrm{AS}^{N}$. It is also stable. Note that $\{a, c\}$ is conflict-free in $\mathrm{AS}^{N}$. Nevertheless $\{a, c\}$ is a conflicting set in the sense of CFS.

\subsection{Comparison between the different frameworks}

In the previous sections, starting from a set of constraints, several frameworks (GBAS, MAS, $\mathrm{AS}^{N}$ and $\mathrm{AS}^{N^{+}}$) have been proposed for handling necessary support. In this section, we compare these frameworks wrt two different points of view: the satisfaction of the constraints and the extensions that are produced.

First, the following table synthesizes the previous results: 


\begin{tabular}{||c||c|c|c|c||}
\cline { 2 - 5 } \multicolumn{1}{c|}{} & GBAS & MAS & AS $^{N}$ & AS $^{N^{+}}$ \\
\hline \hline TRA & $X$ & $X$ & $X$ & $X$ \\
\hline CLO & $X$ & $X$ & $X$ & $X$ \\
\hline CFS & $X$ & - & - & $X$ \\
\hline nATT & - & - & $X$ & $X$ \\
\hline n+ATT & - & - & - & $X$ \\
\hline \hline
\end{tabular}

$X$ (resp. -) means that the corresponding property is (resp. not) satisfied in the corresponding framework.

Now, let us consider $\mathrm{AS}^{N}$ and GBAS. We know that $\mathrm{AS}^{N}$ does not satisfy CFS whereas GBAS does. However, due to Prop. 7, if $S$ is a conflicting set of GBAS, it is conflicting in $\mathrm{AS}^{N^{+}}$and then there is no admissible set of $\mathrm{AS}^{N}$ containing $S$. Moreover, it can be proved that each preferred extension of GBAS is (generally strictly) included in a preferred extension of $\mathrm{AS}^{N}$. This is illustrated by the following example.

Ex. 6 Consider BAS represented by:

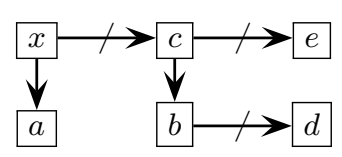

In the associated GBAS, we have $\mathbf{C}=\{\{x, c\},\{x, b\},\{c, e\}$, $\{b, d\},\{a, c\},\{b, e\}\}$. The unique preferred extension of GBAS is $\{a, x, e\}$. In the associated $\mathrm{AS}^{N}$, the $\mathrm{n}$-attacks from $x$ to $b$ is used for ensuring the acceptability of $d$ wrt $\{a, x, e\}$. So, the unique preferred extension is $\{a, d, x, e\}$.

Prop. 13 Let BAS $=\left\langle\mathbf{A}, \mathbf{R}_{\text {att }}, \mathbf{R}_{\text {sup }}\right\rangle$ and its associated GBAS and $\mathrm{AS}^{N}$. Let $S \subseteq \mathbf{A}$. If $S$ is admissible in GBAS, then $S$ is also admissible in $\mathrm{AS}^{N}$. If $S$ is a preferred extension in GBAS, then $S$ is included in a preferred extension of $\mathrm{AS}^{N}$. If $S$ is a stable extension in GBAS, then $S$ is also a stable extension of $\mathrm{AS}^{N}$.

Note that Prop. 13 holds when considering $\mathrm{AS}^{N^{+}}$instead of $\mathrm{AS}^{N}$, due to Prop. 8, 9 and 10.

The next issue concerns the comparison between $\mathrm{AS}^{N}$ and the associated MAS of BAS. It seems that encoding a necessary support $c \mathbf{R}_{\text {sup }} b$ by a metaargument $N_{c b}$ and the sequence $a \mathbf{R}_{\text {att }} c \mathbf{R}_{\text {att }} N_{c b} \mathbf{R}_{\text {att }} b$ is less strong than encoding $\mathrm{n}$-attacks. However, there is a correspondence between the extensions which are obtained in each framework.

Prop. 14 Let $\mathrm{BAS}=\left\langle\mathbf{A}, \mathbf{R}_{\text {att }}, \mathbf{R}_{\text {sup }}\right\rangle$ and its associated MAS and $\mathrm{AS}^{N}$.

- Let $S \subseteq \mathbf{A} \cup \mathbf{A}_{n}$. If $S$ is admissible in MAS, then $S \cap \mathbf{A}$ is also admissible in $\mathrm{AS}^{N}$. If $S$ is stable in MAS, then $S \cap \mathbf{A}$ is also stable in $\mathrm{AS}^{N}$.

- Let $S \subseteq \mathbf{A}$. If $S$ is a preferred extension in $\mathrm{AS}^{N}$, there exists $S^{\prime}$ admissible in MAS such that $S=S^{\prime} \cap \mathbf{A}$. If $S$ is a stable extension in $\mathrm{AS}^{N}$, then there exists $S^{\prime}$ stable in MAS such that $S=S^{\prime} \cap \mathbf{A}$.

From Prop. 13 and 14, the following comparison between GBAS and MAS can be easily established.

Prop. 15 Let $\mathrm{BAS}=\left\langle\mathbf{A}, \mathbf{R}_{\mathrm{att}}, \mathbf{R}_{\text {sup }}\right\rangle$ and its associated MAS and GBAS. Let $S \subseteq \mathbf{A}$. If $S$ is a preferred extension of GBAS, then there exists $S^{\prime}$ preferred in MAS such that $S \subseteq S^{\prime} \cap \mathbf{A}$. If $S$ is a stable extension of GBAS, then there exists $S^{\prime}$ stable in MAS such that $S=S^{\prime} \cap \mathbf{A}$. 
The following example illustrates the above propositions.

Ex.6 (cont'd) Consider the associated MAS represented by:

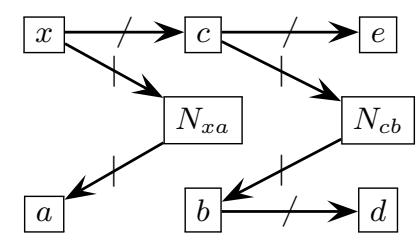
In GBAS, the unique preferred (and also stable) extension is the set $\{a, x, e\}$. In $\mathrm{AS}^{N}$, the unique preferred (and also stable) extension is the set $\{a, x, e, d\}$. In MAS, the unique preferred (and also stable) extension is the set $\left\{a, x, e, N_{c b}, d\right\}$.

\section{Conclusion and future works}

Recent studies in argumentation have addressed the notion of support, with several interpretations (such as deductive, evidential, necessary, backing) and several approaches developed independently. In this paper we focus on necessary support and show that the intended meaning of necessary support can induce different ways to handle it. Our main contribution is to propose an axiomatic approach that is helpful for understanding and comparing the different existing proposals for handling support. First, we have proposed different kinds of constraints that should be imposed to a bipolar argumentation system using necessary supports. Then we have studied different frameworks suitable for encoding these contraints.

This paper reports a preliminary work that could be pursued along different lines. First, our study must be deepened in order to give a more high-level analysis and comparison of all these frameworks. Then the axiomatic approach could be enriched by considering other constraints, such as for instance the strong requirement leading to the addition of symmetric attacks in the case of a necessary support. Moreover, it would be interesting to define such an axiomatic for other interpretations of support, or to consider other frameworks which do not explicitely define a notion of support, such as Abstract Dialectical Frameworks [6]. Another direction for further research would be to study how to encode necessary (or other variants) support by the addition of attacks of various strengths (see for instance $[19 ; 7 ; 16 ; 17]$ ). Moreover it would be interesting to see the link between our approaches and the ranking semantics proposed by [1].

\section{References}

1. Amgoud, L., Ben-Naim, J.: Ranking-based semantics for argumentation frameworks. In: Proc. of SUM, LNCS, vol. 8078, pp. 134-147. Springer (2013)

2. Amgoud, L., Cayrol, C.: A reasoning model based on the production of acceptable arguments. Annals of Mathematics and Artificial Intelligence 34, 197-216 (2002)

3. Amgoud, L., Cayrol, C., Lagasquie-Schiex, M.C., Livet, P.: On bipolarity in argumentation frameworks. Intl. Journal of Intelligent Systems 23, 1062-1093 (2008)

4. Amgoud, L., Maudet, N., Parsons, S.: Modelling dialogues using argumentation. In: Proc. of ICMAS. pp. 31-38 (2000)

5. Boella, G., Gabbay, D.M., van der Torre, L., Villata, S.: Support in abstract argumentation. In: Proc. of COMMA. pp. 111-122. IOS Press (2010) 
6. Brewka, G., Woltran, S.: Abstract dialectical frameworks. In: Proc. of KR. pp. 102-111 (2010)

7. Cayrol, C., Devred, C., Lagasquie-Schiex, M.C.: Acceptability semantics accounting for strength of attacks in argumentation. In: Proc. of ECAI. pp. 995-996 (2010)

8. Cayrol, C., Lagasquie-Schiex, M.C.: On the acceptability of arguments in bipolar argumentation frameworks. In: Proc. of ECSQARU. pp. 378-389 (2005)

9. Cayrol, C., Lagasquie-Schiex, M.C.: Coalitions of arguments: a tool for handling bipolar argumentation frameworks. Intl. J. of Intelligent Systems 25, 83-109 (2010)

10. Cayrol, C., Lagasquie-Schiex, M.C.: Bipolarity in argumentation graphs: towards a better understanding. IJAR 54(7), 876-899 (2013)

11. Cayrol, C., Lagasquie-Schiex, M.C.: An axiomatic approach to support in argumentation. Tech. Rep. RR-2015-04-FR, IRIT (2015), http://www.irit.fr/publis/ ADRIA/PapersMCL/Rapport-IRIT-2015-04.pdf

12. Cohen, A., Gottifredi, S., García, A.J., Simari, G.R.: An approach to abstract argumentation with recursive attack and support. Journal of Applied Logic (2014)

13. Cohen, A., Gottifredi, S., García, A.J., Simari, G.R.: A survey of different approaches to support in argumentation systems. The Knowledge Engineering Review 29, 513-550 (2014)

14. Coste-Marquis, S., Devred, C., Marquis, P.: Symmetric argumentation frameworks. In: Proceedings of ECSQARU, LNAI 3571. pp. 317-328. Springer-Verlag (2005)

15. Dung, P.M.: On the acceptability of arguments and its fundamental role in nonmonotonic reasoning, logic programming and n-person games. Artificial Intelligence 77, 321-357 (1995)

16. Dunne, P., Hunter, A., McBurney, P., Parsons, S., Wooldridge, M.: Weighted argument systems: Basic definitions, algorithms, and complexity results. Artificial Intelligence $175(2), 457-486$ (2011)

17. Kaci, S., Labreuche, C.: Arguing with valued preference relations. In: Proc. of ECSQARU. pp. 62-73 (2011)

18. Karacapilidis, N., Papadias, D.: Computer supported argumentation and collaborative decision making: the HERMES system. Information systems 26(4), 259-277 (2001)

19. Martinez, D.C., Garcia, A.J., Simari, G.R.: An abstract argumentation framework with varied-strength attacks. In: Proc of KR. pp. 135-143 (2008)

20. Nouioua, F.: AFs with necessities: further semantics and labelling characterization. In: Proc. of SUM (2013)

21. Nouioua, F., Risch, V.: Bipolar argumentation frameworks with specialized supports. In: Proc. of ICTAI. pp. 215-218. IEEE Computer Society (2010)

22. Nouioua, F., Risch, V.: Argumentation frameworks with necessities. In: Proc. of SUM. pp. 163-176. Springer-Verlag (2011)

23. Oren, N., Norman, T.J.: Semantics for evidence-based argumentation. In: Proc. of COMMA. pp. 276-284 (2008)

24. Oren, N., Reed, C., Luck, M.: Moving between argumentation frameworks. In: Proc. of COMMA. pp. 379-390. IOS Press (2010)

25. Polberg, S., Oren, N.: Revisiting support in abstract argumentation systems. In: Proc. of COMMA. pp. 369-376. IOS Press (2014)

26. Prakken, H.: On support relations in abstract argumentation as abstraction of inferential relations. In: Proc. of ECAI. pp. 735-740 (2014)

27. Verheij, B.: Deflog: on the logical interpretation of prima facie justified assumptions. Journal of Logic in Computation 13, 319-346 (2003)

28. Villata, S., Boella, G., Gabbay, D.M., van der Torre, L.: Modelling defeasible and prioritized support in bipolar argumentation. AMAI 66(1-4), 163-197 (2012) 